\title{
Experimental demonstration of continuous variable cloning with phase-conjugate inputs
}

Sabuncu, Metin; Andersen, Ulrik Lund; Leuchs, G.

Published in:

Physical Review Letters

Link to article, DOI:

10.1103/PhysRevLett.98.170503

Publication date:

2007

Document Version

Publisher's PDF, also known as Version of record

Link back to DTU Orbit

Citation (APA):

Sabuncu, M., Andersen, U. L., \& Leuchs, G. (2007). Experimental demonstration of continuous variable cloning with phase-conjugate inputs. Physical Review Letters, 98(17), 170503.

https://doi.org/10.1103/PhysRevLett.98.170503

\section{General rights}

Copyright and moral rights for the publications made accessible in the public portal are retained by the authors and/or other copyright owners and it is a condition of accessing publications that users recognise and abide by the legal requirements associated with these rights.

- Users may download and print one copy of any publication from the public portal for the purpose of private study or research.

- You may not further distribute the material or use it for any profit-making activity or commercial gain

- You may freely distribute the URL identifying the publication in the public portal

If you believe that this document breaches copyright please contact us providing details, and we will remove access to the work immediately and investigate your claim 


\title{
Experimental Demonstration of Continuous Variable Cloning with Phase-Conjugate Inputs
}

\author{
Metin Sabuncu, ${ }^{1,2}$ Ulrik L. Andersen, ${ }^{1,2}$ and Gerd Leuchs ${ }^{2}$ \\ ${ }^{1}$ Department of Physics, Technical University of Denmark, Building 309, 2800 Kgs. Lyngby, Denmark \\ ${ }^{2}$ Institut für Optik, Information und Photonik, Max-Planck Forschungsgruppe, Universität Erlangen-Nürnberg, \\ Günther-Scharowsky Strasse 1, 91058, Erlangen, Germany \\ (Received 7 December 2006; published 24 April 2007)
}

\begin{abstract}
We report the first experimental demonstration of continuous variable cloning of phase-conjugate coherent states as proposed by Cerf and Iblisdir [Phys. Rev. Lett. 87, 247903 (2001)]. In contrast to this proposal, the cloning transformation is accomplished using only linear optical components, homodyne detection, and feedforward. As a result of combining phase conjugation with a joint measurement strategy, superior cloning is demonstrated with cloning fidelities reaching $89 \%$.
\end{abstract}

\section{DOI: 10.1103/PhysRevLett.98.170503}

One of the most intriguing results of quantum mechanics is that an unknown quantum state cannot be exactly cloned $[1,2]$. This fact stems from the inherent linearity of quantum mechanics, and is one of the most discussed features in recent years because it enables secure quantum communication such as quantum key distribution and secret sharing $[3,4]$. Because quantum cloning is, in general, imperfect one is led to the construction of optimal, but imperfect, quantum cloning machines. Such machines based on qubits have been experimentally realized in several different settings. On the other hand, cloning machines based on continuous variables have only very recently been implemented $[5,6]$.

Yet another interesting feature of quantum mechanics was discovered in 1999 by Gisin and Popescu [7]. They predicted that more quantum information can be encoded into pairs of antiparallel spins than in parallel ones. The continuous variable (CV) analogue of this effect was addressed theoretically by Cerf and Iblisdir who showed that more CV quantum information can be encoded into pairs of phase-conjugate coherent states, $|\alpha\rangle\left|\alpha^{*}\right\rangle$, than in pairs of identical coherent states, $|\alpha\rangle|\alpha\rangle$ [8]. Because of the existence of a strong link between cloning and measurement theory, the superiority of using antiparallel spins or phaseconjugate coherent states led Cerf and Iblisdir to suggest that cloning machines with such inputs perform better than conventional cloning machines. This was indeed the case as shown theoretically in Ref. [9] for phase-conjugate coherent states and in Ref. [10] for antiparallel spin states. Recently, these results were generalized to $d$-dimensional systems [11]. Cerf and Iblisdir also suggested to realize the physical implementation of the cloning of phase-conjugate input states by a sequence of beam splitters, a nonlinear process, and another sequence of beam splitters [9].

In this Letter we report the first experiment demonstrating superior performance of a quantum protocol using a pair of phase-conjugate states. In particular, we are proposing and implementing a novel approach for phaseconjugate cloning which is not relying on a nonlinear parametric process. A simple combination of beam splitters, detectors, and feedforwards suffices to enable optimal
PACS numbers: 03.67.Hk, 03.65.Ta, 42.50.Dv, 42.50.Lc

$N+N \rightarrow M$ Gaussian cloning with phase-conjugate input states, where $N$ replicas of $|\alpha\rangle$ and $N$ replicas of $\left|\alpha^{*}\right\rangle$ serve as inputs to produce $M$ clones. Theoretically, we treat the general case of $N+N \rightarrow M$ cloning while the $1+1 \rightarrow 2$ and $1+1 \rightarrow 3$ cloning of phase-conjugate inputs are experimentally demonstrated. We note that the cloning protocol with phase-conjugate inputs realized in this Letter has never been implemented before in any quantum system. It is, to the best of our knowledge, the first example of a continuous variable quantum information processing experiment for which there is no experiment with discrete variables.

The figure of merit normally used to quantify the quality of a cloning transformation is the average fidelity, which is a measure of the similarity between the input states and the clones. When considering a flat distribution of coherent states as inputs and assuming that the cloning transformation conserves the Gaussian statistics of the quadratures, the optimal $2 N \rightarrow M$ cloning machine yields the average fidelity [12]

$$
F_{C}=\frac{2 M N}{2 M N+M-2 N} \text {. }
$$

On the other hand, it has been found that the optimal $N+$ $N \rightarrow M$ cloning machine with phase-conjugate input states produces clones with fidelity $[9,13]$

$$
F_{\mathrm{PC}}=\frac{4 M^{2} N}{4 M^{2} N+(M-N)^{2}} .
$$

Here $2 N$ corresponds to the total number of inputs consisting either of $2 N$ replicas of $|\alpha\rangle$ in Eq. (1) or of $N$ replicas of $|\alpha\rangle$ and $N$ replicas of $\left|\alpha^{*}\right\rangle$ in Eq. (2). The difference of the two fidelities for $N=1$ and various numbers of the outputs is depicted in the inset of Fig. 1, and it is clearly seen that the phase-conjugate cloning machine outperforms the conventional one for $M>2$.

A schematic of the cloning machine proposed in this Letter is presented in Fig. 1. The input signal is contained in an ensemble consisting of $N$ identical coherent states and $N$ identical phase-conjugate coherent states. The two sets of states are uniquely described by the relations 


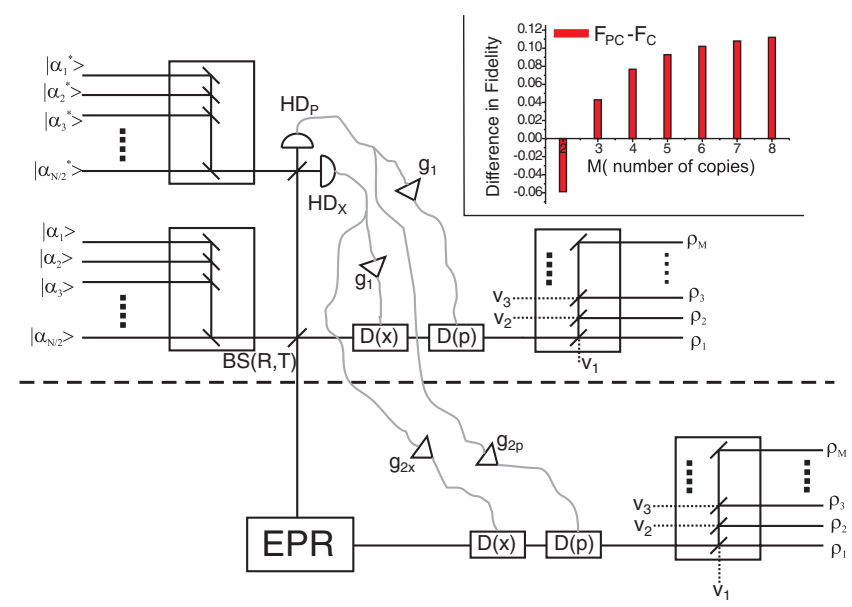

FIG. 1 (color online). Proposed setup for an $N+N \rightarrow M$ cloning machine. $\mathrm{BS}(T)$ : Variable beam splitter with transmission $T$ and reflection $R$. $\mathrm{HD}_{x(p)}$ : Homodyne detector measuring $x(p) . g_{1}, g_{2 x}$, and $g_{2 p}$ : Electronic gains. $D(x, p)$ : Displacers of $x$ and $p$. EPR: Einstein-Podolsky-Rosen entanglement source. $v_{i}$ denotes the vacuum inputs while $\rho_{i}$ denotes the density operators of the outputs.

$|\alpha\rangle^{\otimes N}=|x+i p\rangle^{\otimes N}$ and $\left|\alpha^{*}\right\rangle^{\otimes N}=|x-i p\rangle^{\otimes N}$, where $x$ is the amplitude quadrature, $p$ is the phase quadrature, and $[x, p]=2 i$. Each of the two sets of states are then collected into two single states using two arrays of $N-1$ beam splitters. In the Heisenberg picture, the amplitude quadratures after collection can be written as

$$
\begin{aligned}
& \hat{x}_{c 1}=\frac{1}{\sqrt{N}} \sum_{k=1}^{N} \hat{x}_{k}=\frac{1}{\sqrt{N}}\left(N\langle\hat{x}\rangle+\sum_{k=1}^{N} \delta \hat{x}_{k}\right), \\
& \hat{x}_{c 2}=\frac{1}{\sqrt{N}} \sum_{l=1}^{N} \hat{x}_{l}^{\prime}=\frac{1}{\sqrt{N}}\left(N\langle\hat{x}\rangle+\sum_{l=1}^{N} \delta \hat{x}_{l}^{\prime}\right),
\end{aligned}
$$

where $\hat{x}_{k, l}$ have been decomposed as $\hat{x}_{k, l}=\langle\hat{x}\rangle+\delta \hat{x}_{k, l}$ and $\langle\hat{x}\rangle=\left\langle\hat{x}_{k}\right\rangle=\left\langle\hat{x}_{l}\right\rangle$. The collective coherent state impinges on a beam splitter (with transmission $T$ and reflection $1-$ $T$ ) and the reflected part is measured jointly with the collective phase conjugated coherent state using a symmetric beam splitter and two homodyne detectors measuring $\hat{x}$ and $\hat{p}$. The measurement outcomes are electronically amplified with gain $g$ and used to displace the remaining part of the collective coherent state. Such a combination of linear optics, measurements, and feedforward enables a quantum noise limited amplification if $g_{1}=\sqrt{2(1-T) / T}$, and the input-output relation is simply $\hat{x}_{\text {out }}=\sqrt{1 / T} \hat{x}_{c 1}+$ $\sqrt{(1-T) / T} \hat{x}_{c 2}$ [14]. Finally, the resulting state is divided into $M$ clones using an $M$ splitter. The relation between the inputs and any output clone is thus

$$
\begin{aligned}
\hat{x}_{i}= & \frac{1}{\sqrt{T N M}}\left((N+N \sqrt{1-T})\langle\hat{x}\rangle+\sum_{k=1}^{N} \delta \hat{x}_{k}\right. \\
& \left.+\sqrt{1-T} \sum_{l=1}^{N} \delta \hat{x}_{l}^{\prime}\right)+\sum_{j=1}^{M-1} \kappa_{i j} \hat{x}_{v j},
\end{aligned}
$$

where $\hat{x}_{v j}$ represents vacuum fluctuations and $\kappa_{i j}$ are coefficients that depend on $M$. Universal cloning, that is, cloning with conservation of the mean value of $\hat{x}$, is obtained for $T=4 M N /(M+N)^{2}$. In that case the variance of the amplitude quadrature noise of any clone is

$$
\Delta^{2} \hat{x}_{i}=1+\frac{(M-N)^{2}}{2 M^{2} N} .
$$

The same analysis applies for the phase quadrature and thus it is readily verified that the cloning transformation is symmetric in $\hat{x}$ and $\hat{p}: \Delta^{2} \hat{p}_{i}=\Delta^{2} \hat{x}_{i}$. Now, by using the relation for universal cloning fidelity of coherent states

$$
F=\frac{2}{\sqrt{\left(1+\bar{\Delta}^{2} \hat{x}_{i}\right)\left(1+\bar{\Delta}^{2} \hat{p}_{i}\right)}},
$$

we immediately retain the optimal fidelity in (2). Hence, optimal Gaussian cloning of phase-conjugate coherent states can be obtained using simple linear optics, homodyne detection, and feedforward.

We note that the presented cloning machine is nonunitary. To ensure unitarity, an Einstein-Podolsky-Rosen (EPR) entangled resource must be applied as shown below the dashed line in Fig. 1: One half of the EPR state is injected into the beam splitter $\operatorname{BS}(T)$ while the second half is displaced according to the measurement outcomes of the measurements (scaled with gains $g_{2 x}=\sqrt{2 / T}$ and $g_{2 p}=$ $-\sqrt{2 / T})$. The latter half is subsequently divided into $M$ clones of $\left|\alpha^{*}\right\rangle$. Therefore this unitary cloning machine produces not only $M$ optimal coherent state clones but also $M$ optimal phase-conjugate coherent state clones.

We now proceed with the experimental demonstration of the production of two and three clones from a single pair of phase-conjugate coherent states. The experimental setup is shown in Fig. 2. As a laser source we used a Nd:YAG laser emitting light at $1064 \mathrm{~nm}$. The laser beam was split into three parts: two parts served as input modes whereas the last part was used as auxiliary and local oscillator beams. Coherent states are generated at sidebands of laser modes. These sidebands, originally in the vacuum state, are excited by the use of amplitude and phase modulators driven by a signal generator. One of the phase modulators is driven $\pi$ out of phase with respect to the other one to ensure the production of phase-conjugate beams. In contrast, the amplitude modulators are driven in phase. The phase relation

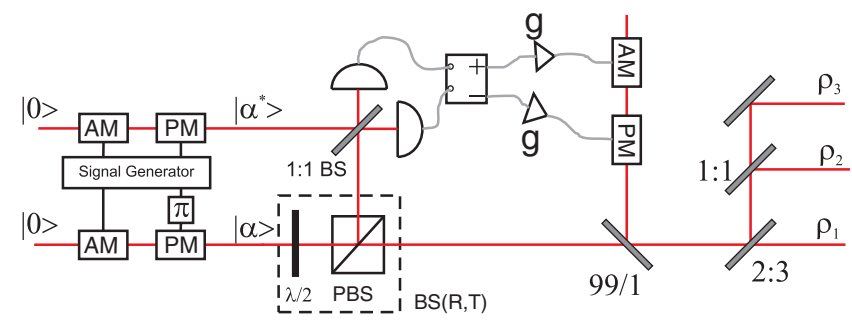

FIG. 2 (color online). Schematic of the experimental setup. AM: amplitude modulator; PM: phase modulator; $g$ : electronic gains. $\rho_{1}, \rho_{2}$, and $\rho_{3}$ are the density operators of the clones. 
between the two input states is verified by interfering the two states on a 50/50 beam splitter and subsequently measuring the amplitude and phase quadratures in the two outputs of the beam splitter. Extinction of the amplitude (phase) quadrature in the difference (sum) output port of the beam splitter is a clear signature of the preparation of states with proper phase relation and identical amplitudes.

After preparation of the pair of phase-conjugate coherent states $\left(|\alpha\rangle\right.$ and $\left.\left|\alpha^{*}\right\rangle\right)$, they are injected into the cloning machine. A tunable beam splitter, consisting of a half wave plate and a polarizing beam splitter, separates the coherent state, $|\alpha\rangle$, into two parts. For the production of two or three clones the transmission was set to $T=8 / 9$ and $T=3 / 4$, respectively, in order to optimize the cloning fidelity. The reflected part of the input state interferes with the phaseconjugate state, $\left|\alpha^{*}\right\rangle$, on a balanced beam splitter. The carrier power of the input modes has been tailored such that the powers of the two states in the joint measurement are balanced. This enables the joint measurements of $x$ and $p$ using the simplified setup shown in Fig. 2: After interference at the beam splitter with a $\pi / 2$ relative phase shift, the outputs are directly detected. Subsequently, the sum and difference currents are produced, which represent the sum of amplitude quadratures and the difference of the phase quadratures of the two inputs to the beam splitter. The electronic gains in the feedforward loop are adjusted to ensure close to unity cloning gains. The scaled measurement outcomes are used to modulate an auxiliary beam which subsequently is coupled with the remaining part of $|\alpha\rangle$ using a very asymmetric beam splitter (with splitting ratio 99/1). This accomplishes the displacement operation, and finally the two or three clones are produced by using a single symmetric beam splitter (not shown in Fig. 2) or two beam splitters with ratios 2:3 and 1:1.

Knowing that the Wigner functions of the inputs and the outputs are Gaussian, they are fully characterized by measuring the first and second order moments of $x$ and $p$. This is done by using homodyne detection where the local oscillator is stably locked for accessing either $x$ or $p$. The mean and variance at the sideband were analyzed using a spectrum analyzer which selects the frequency of 14.3 MHz with a resolution bandwidth of $100 \mathrm{kHz}$.

In order to determine accurately the optical gains for the various clones, we measured the signal power of the input and output. By comparing these results we estimate the gains, the exact values of which can be found in the caption text of Figs. 3 and 4. In the following we assume the gains to be unity and consider later implications of nonunity gains. After the gain measurements, the input modulators were switched off in order to precisely measure the cloning noise at $14.3 \mathrm{MHz}$ and thus quantify the cloning performance. Typical examples of measurement runs for the production of two and three clones are shown in Figs. 3 and 4, respectively. Here the added cloning noises relative to the shot noise of the input state for the amplitude and phase quadratures are displayed. From these measurements and employing Eq. (7) we can easily determine the cloning
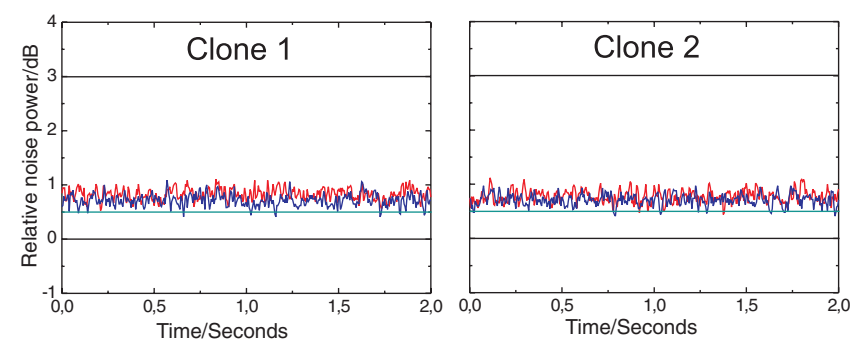

FIG. 3 (color online). Spectral noise powers relative to the shot noise for the two clones. Red (light gray) and blue (dark gray) traces correspond to the added cloning noise with respect to the input state for the phase and amplitude quadrature, respectively. The $1 \rightarrow 2,1+1 \rightarrow 2$, and $2 \rightarrow 2$ cloning limits are illustrated by solid straight lines at the $3,0.5$, and $0 \mathrm{~dB}$ levels. The data were corrected taking into account the homodyne detection efficiencies which were measured to be $83 \%$ and $85 \%$ for clones 1 and 2 . The noises were measured to $\Delta^{2} x_{1}=1.15 \pm 0.2$ and $\Delta^{2} p_{1}=$ $1.18 \pm 0.2$ for clone 1 , and $\Delta^{2} x_{2}=1.19 \pm 0.2$ and $\Delta^{2} p_{2}=$ $1.19 \pm 0.2$ for clone 2. The optical gains for this particular measurement run were $G_{p 1}=1.01 \pm 0.01, G_{x 1}=1.02 \pm 0.01$, $G_{p 2}=1.00 \pm 0.01$, and $G_{x 2}=0.99 \pm 0.01$. Video bandwidth $30 \mathrm{~Hz}$ and sweep time $2 \mathrm{sec}$.

fidelities. For the $1+1 \rightarrow 2$ cloning operation the copies are produced with fidelities $92.4 \% \pm 1 \%$ and $91.3 \% \pm$ $1 \%$, whereas the $1+1 \rightarrow 3$ cloning machine produces copies with fidelities $88.3 \% \pm 1 \%, 88.9 \% \pm 1 \%$, and $88.7 \% \pm 1 \%$.

The latter values demonstrate that the $1+1 \rightarrow 3$ cloning machine with phase-conjugate inputs operates very close to the optimum of $90 \%$ [Eq. (2)] and it outperforms the conventional cloning machine which ideally yields a fidelity of $85.7 \%$ [Eq. (1)]. The close to optimal performance is a result of the high quality of the feedforward loop. The quantum efficiencies of the detectors (including the interference visibility) were measured to $93 \%$ and the electronic noise was negligible. The $1+1 \rightarrow 2$ cloning fidelities are of course not surpassing the fidelity for the conventional $2 \rightarrow 2$ cloning machine which trivially yields a fidelity of $100 \%$. However, using phase-conjugate inputs, there exists also the possibility of producing optimal copies of $\left|\alpha^{*}\right\rangle$ in addition to copies of $|\alpha\rangle$, which is not possible with the conventional cloning approach.

In the fidelity calculations we assumed unity gains. There was, however, a small deviation from unity, and thus strictly speaking the cloning machine is not universal with respect to the flat coherent state alphabet. The set of coherent states must be restricted, and the corresponding average fidelity must be computed. Assuming that the input alphabet is restricted to a Gausssian distribution with variance equal to 10 vacuum units, the average fidelities can be determined to $0.87,0.87$, and 0.89 for the $1+$ $1 \rightarrow 3$ cloning machine and 0.92 and 0.92 for the $1+1 \rightarrow$ 2 cloning machine [15].

We have now experimentally proved the surprising fact that a cloning machine with phase-conjugate input states 

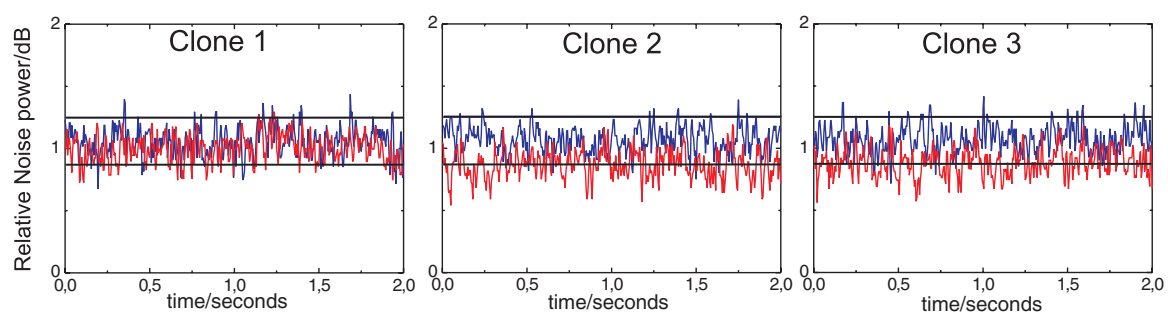

FIG. 4 (color online). Spectral noise powers relative to the shot noise for the three clones. The straight lines at the 1.25 and $0.87 \mathrm{~dB}$ level represent the optimal limits for $2 \rightarrow 3$ and $1+1 \rightarrow 3$ cloning, respectively. The data were corrected taking into account the homodyne detection efficiencies which were measured to be $83 \%, 85 \%$, and $80 \%$ for clones 1, 2, and 3. For this particular measurement run we measured the following noises: $\Delta^{2} x_{1}=1.26 \pm 0.2, \Delta^{2} p_{1}=1.27 \pm 0.2, \Delta^{2} x_{2}=1.22 \pm 0.2, \Delta^{2} p_{2}=1.28 \pm$ $0.2, \Delta^{2} x_{3}=1.23 \pm 0.2$, and $\Delta^{2} p_{3}=1.28 \pm 0.2$; and the following gains: $G_{p 1}=1.00 \pm 0.01, G_{x 1}=0.95 \pm 0.01, G_{p 2}=0.95 \pm$ $0.01, G_{x 2}=0.96 \pm 0.01, G_{p 3}=1.01 \pm 0.01$, and $G_{x 3}=1.00 \pm 0.01$.

performs better than a cloning machine with identical inputs. The fact that a pair of phase-conjugate coherent states is more informative than identical ones led to a suppression of the noise induced by the cloning action. This close relation between the cloning noise and the information content of the input states is easily understood from the part of the setup executing a joint measurement of phase conjugated coherent states. Such a measurement strategy has recently been proven to be superior for information retrieval of phase-conjugate states [16]. In contrast, for identical coherent states such nonlocal measurement strategy has no advantage over the standard local strategy. Thus the phase conjugation combined with the joint measurement strategy yields more information which in turn leads to less noise in the displacement operation and subsequently less noise added to the clones.

It is also clear from the setup that the production of infinitely many clones $(M \rightarrow \infty)$ coincides with optimal estimation as proved by Bae and Acin [17]. For $M \rightarrow \infty$, the transmission $T \rightarrow 0$, which results in a complete joint measurement of the phase-conjugate coherent states, where $|\alpha\rangle$ and $\left|\alpha^{*}\right\rangle$ interfere at a 1:1 beam splitter and conjugate quadratures are measured. This measurement strategy coincides with the optimal one for estimating the information in phase-conjugate coherent states [16], thus illustrating the strong link between cloning and measurement theory.

Finally, we mention that the combined use of phase conjugation and joint measurement for improving the performance of a quantum protocol has many applications beyond the cloning action. Recently it was realized that such a strategy can be used to execute a minimum disturbance measurement [18], enable an optimal individual eavesdropping attack [19], and increase the sensitivity of optical coherence tomography beyond the coherent state limit and ultimately reaching the entanglement based limit [20].

We thank R. Filip for stimulating discussions. This work has been supported by the EU project COVAQIAL (Project No. FP6-511004).
Note added in proof. - A similar proposal, but without an experimental verification, has recently been published [21].

[1] W. K. Wootters and W. H. Zurek, Nature (London) 299, 802 (1982).

[2] D. Dieks, Phys. Lett. A 92, 271 (1982).

[3] V. Scarani et al., Rev. Mod. Phys. 77, 1225 (2005).

[4] N. J. Cerf and J. Fiurasek, in Progress in Optics, edited by E. Wolf (Elsevier, Amsterdam, 2006), Vol. 49, p. 455.

[5] U. L. Andersen, V. Josse, and G. Leuchs, Phys. Rev. Lett. 94, 240503 (2005).

[6] S. Koike et al., Phys. Rev. Lett. 96, 060504 (2006).

[7] N. Gisin and S. Popescu, Phys. Rev. Lett. 83, 432 (1999).

[8] N. J. Cerf and S. Iblisdir, Phys. Rev. A 64, 032307 (2001).

[9] N. J. Cerf and S. Iblisdir, Phys. Rev. Lett. 87, 247903 (2001).

[10] J. Fiurasek et al., Phys. Rev. A 65, 040302 (2002).

[11] X.-F. Zhou, Y.-S. Zhang, and G.-C. Guo, Phys. Rev. A 74, 042324 (2006).

[12] N. Cerf and S. Iblisdir, Phys. Rev. A 62, 040301(R) (2000).

[13] We note that non-Gaussian operations improve the standard cloning protocol [N. Cerf et al., Phys. Rev. Lett. 95, 070501 (2005)], and we conjecture this also to be the case for cloning with phase conjugated inputs.

[14] V. Josse et al., Phys. Rev. Lett. 96, 163602 (2006).

[15] P. T. Cochrane, T. C. Ralph, and A. Doliska, Phys. Rev. A 69, 042313 (2004).

[16] J. Niset et al., quant-ph/0608215.

[17] J. Bae and A. Acin, Phys. Rev. Lett. 97, 030402 (2006).

[18] U.L. Andersen et al., Phys. Rev. Lett. 96, 020409 (2006).

[19] R. Namiki, M. Koashi, and N. Imoto, Phys. Rev. A 73, 032302 (2006).

[20] B. I. Erkmen and J. H. Shapiro, Phys. Rev. A 74, 041601 (2006).

[21] H. Chen and J. Zhang, Phys. Rev. A 75, 022306 (2007). 